\title{
Can plant-soil feedback link genetic diversity and species diversity?
}

\author{
Lana Bolin ${ }^{1}$ and Jennifer Lau ${ }^{1}$ \\ ${ }^{1}$ Indiana University Bloomington
}

September 25, 2020

\begin{abstract}
Genetic diversity and species diversity are typically studied in isolation despite theory showing they likely influence one another. Here, we used simplified communities of one or two populations of one or two species to test whether linkages between genetic and species diversity can be mediated by interactions between plants and their soil microbiota, or microbe-mediated plant-soil feedback (PSF). Interspecific PSF promotes the maintenance of species diversity when plants grow better with heterospecific soil microbes than with conspecific microbes. Similarly, intraspecific PSF promotes the maintenance of genetic diversity when plants grow better with heterogenotypic than with congenotypic microbes. We found that genetic diversity reduced the ability of interspecific PSF to maintain species diversity, and, for one study species, species diversity reduced the ability of intraspecific PSF to maintain genetic diversity. If these patterns occur in more diverse communities, then PSF may cause genetic and species diversity to negatively influence one another.
\end{abstract}

\section{Hosted file}

Bolin_PSF_EcolLett_resubmission.pdf available at https://authorea.com/users/361861/articles/ 483157-can-plant-soil-feedback-link-genetic-diversity-and-species-diversity 